\title{
Multidimensional Lobachevsky Spline Integration on Scattered Data
}

\author{
Giampietro Allasia, Roberto Cavoretto* and Alessandra De Rossi \\ Department of Mathematics "G. Peano”, University of Torino, Via Carlo Alberto 10, 10123 Torino, Italy
}

Received: 4 Jun. 2013, Revised: 18 Sep. 2013, Accepted: 19 Sep. 2013

Published online: 1 Jan. 2014

\begin{abstract}
This paper deals with the topic of numerical integration on scattered data in $\mathbb{R}^{d}, d \leq 10$, by a class of spline functions, called Lobachevsky splines. Precisely, we propose new integration formulas based on Lobachevsky spline interpolants, which take advantage of being expressible in the multivariate setting as a product of univariate integrals. Theoretically, Lobachevsky spline integration formulas have meaning for any $d \in \mathbb{N}$, but numerical results appear quite satisfactory for $d \leq 10$, showing good accuracy and stability. Some comparisons are given with radial Gaussian integration formulas and a quasi-Monte Carlo method using Halton data points sets.
\end{abstract}

Keywords: Lobachevsky splines, scattered data interpolation, integration formulas, high-dimendional integrals, quasi-Monte Carlo methods

\section{Introduction}

The topic of numerical integration on multivariate scattered data has recently gained popularity and interest in various areas of applied mathematics and scientific computing, such as multivariate interpolation, approximation theory, and computer physics (see [7,13, $14,15,19,20,23,24])$, although it has been much less developed with respect to the construction of integration formulas on data with a prefixed distribution (see, e.g., [12]).

In particular, we consider the problem of constructing new integration formulas for high-dimensional scattered data by a class of spline functions, called Lobachevsky splines, arisen in probability theory $[17,21]$ and then also proposed in multivariate interpolation on scattered data [4] and landmark-based image registration [1,3,6]. Lobachevsky splines consist in an infinite sequence of univariate spline functions depending on a shape parameter, which are compactly supported, strictly positive definite, and enjoy noteworthy theoretical and computational properties, such as the convergence to the Gaussian function and the convergence of the sequence of their integrals and derivatives to integrals and derivatives of the Gaussian, respectively (see [4]).

Now, starting from the previous work [5], where we investigate the integration problem on scattered data in
$\mathbb{R}^{d}$, for $d=1,2$, in this paper we focus on the use of Lobachevsky spline integration formulas for $3 \leq d \leq 10$. The idea of using Lobachevsky splines to construct integration formulas on scattered data turns out to be quite natural, not only for the recent interest in meshfree numerical integration, but also for the performance and computation easiness of Lobachevsky spline integrals. In fact, Lobachevsky spline interpolation formulas take advantage of being expressible in the multivariate setting as a product of univariate functions. This makes simple the computation of $d$-dimensional integrals on hypercubes: at first, the integrand function is approximated by a Lobachevsky spline interpolation formula; then, the $d$-variate integral is evaluated as a product of $d$ univariate integrals. The proposed formulas may be interesting because other effective techniques for multidimensional integration on scattered data such as Monte Carlo methods, which are usually used in high dimensions, have poor convergence rates (see e.g. [18, 22]).

Moreover, we remark that Lobachevsky spline interpolation formulas are neither mesh-based formulas (no grid is here considered) nor radial ones, but they asymptotically behave like Gaussian interpolants (see [4]). This feature, together with the uniform convergence of Lobachevsky splines to the Gaussians, allows us to give error estimates for Lobachevsky splines integration

\footnotetext{
*Corresponding author e-mail: roberto.cavoretto@unito.it
} 
formulas. Numerical experiments point out that Lobachevsky spline interpolants are comparable in accuracy with Gaussian interpolants, but they are usually much better conditioned than the Gaussians.

The paper is organized as follows. In Section 2 we consider the problem of scattered data interpolation by Lobachevsky splines, recalling their analytic expressions and some properties. In Section 3 we firstly describe the resulting integration formulas obtained by Lobachevsky spline interpolants, then we analyze conditioning and errors of Lobachevsky spline integration formulas, discussing the role of two essential quantities: the spectral norm of the inverse of the interpolation matrix and the 1-norm of the weight vector. Section 4 summarizes several numerical experiments in order to verify performances of Lobachevsky spline integration formulas, focusing on both accuracy (integration errors) and stability (condition numbers) and comparing Lobachevsky spline results with those of radial Gaussian integration formulas and a quasi-Monte Carlo considering Halton point sets. Finally, Section 5 deals with conclusions and future works.

\section{Lobachevsky spline interpolation}

Let us consider a continuous function $g: \Omega \rightarrow \mathbb{R}$ on a compact domain $\Omega \subset \mathbb{R}^{d}, \quad d \geq 1$, a set $\mathscr{X}=\left\{\mathbf{x}_{i}=\left(x_{1 i}, x_{2 i}, \ldots, x_{d i}\right), i=1,2, \ldots, N\right\} \subset \Omega$ of scattered data points, and the vector $\mathbf{g}=\left\{g\left(\mathbf{x}_{i}\right), i=1,2, \ldots, N\right\}$ of the corresponding function values.

For even $n \geq 2$, we construct the Lobachevsky spline interpolant of $g$ at the nodes $\mathbf{x}_{i}$ in the form

$F_{n}(\mathbf{x})=\sum_{j=1}^{N} c_{j} \phi_{n j}(\mathbf{x}), \quad \mathbf{x} \in \Omega$,

requiring $F_{n}\left(\mathbf{x}_{i}\right)=g\left(\mathbf{x}_{i}\right), i=1,2, \ldots, N$. The interpolant $F_{n}$ is a linear combination of products of univariate shifted and rescaled functions $f_{n}^{*}$

$\phi_{n j}(\mathbf{x}) \equiv \phi_{n j}(\mathbf{x} ; \alpha)=\prod_{h=1}^{d} f_{n}^{*}\left(\alpha\left(x_{h}-x_{h j}\right)\right)$,

where for $j=1,2, \ldots, N$

$$
\begin{aligned}
f_{n}^{*}\left(\alpha\left(x_{h}-x_{h j}\right)\right) & =\sqrt{\frac{n}{3}} \frac{1}{2^{n}(n-1) !} \sum_{k=0}^{n}(-1)^{k}\left(\begin{array}{l}
n \\
k
\end{array}\right) \\
& \times\left[\sqrt{\frac{n}{3}} \alpha\left(x_{h}-x_{h j}\right)+(n-2 k)\right]_{+}^{n-1},
\end{aligned}
$$

and $\alpha \in \mathbb{R}^{+}$is a shape parameter. The coefficients $\mathbf{c}=\left\{c_{j}\right\}$ are computed by solving the linear system

$A \mathbf{c}=\mathbf{g}$,

where the interpolation matrix

$A=\left\{a_{i j}\right\}=\left\{\phi_{n j}\left(\mathbf{x}_{i}\right)\right\}, \quad i, j=1,2, \ldots, N$, is symmetric and depends on the choice of $n$ and $\alpha$ in (2). Since Lobachevsky splines are strictly positive definite for any even $n \geq 2$, the interpolation matrix $A$ in (5) is positive definite for any set of distinct nodes.

From the central limit theorem (see $[17,21]$ ), we remark that the $d$-variate Lobachevsky spline converges for $n \rightarrow \infty$ to the $d$-variate Gaussian, i.e.

$\lim _{n \rightarrow \infty} \prod_{i=1}^{d} f_{n}^{*}\left(\alpha x_{i}\right)=\frac{1}{(2 \pi)^{d / 2}} \exp \left(\frac{-\alpha^{2}\left(\sum_{i=1}^{d} x_{i}^{2}\right)}{2}\right)$.

Hence, Lobachevsky splines asymptotically behave like radial functions, though they are not radial in themselves.

Finally, we observe that, since Lobachevsky splines are (univariate) strictly positive definite functions for even $n \geq$ 2 , we can construct multivariate strictly positive definite functions from univariate ones (see, e.g., [25]), expressing them as products of Lobachevsky splines [4].

\section{Lobachevsky spline integration}

\subsection{Approximation of $d$-variate integrals}

Let us now consider the problem of computing an approximate value of the integral

$I(g)=\int_{\Omega} g(\mathbf{x}) d \mathbf{x}, \quad \mathbf{x} \in \Omega \subset \mathbb{R}^{d}$,

where $g(\mathbf{x})$ is an integrable function, which is generally known only on a scattered data set.

Solving this problem consists in integrating a formula of type (1), which interpolates the given data, that is,

$$
\begin{aligned}
I(g) \approx I\left(F_{n}\right) & =\int_{\Omega} \sum_{j=1}^{N} c_{j} \phi_{n j}(\mathbf{x}) d \mathbf{x} \\
& =\sum_{j=1}^{N} c_{j} \int_{\Omega} \phi_{n j}(\mathbf{x}) d \mathbf{x} \\
& =\sum_{j=1}^{N} c_{j} I\left(\phi_{n j}\right) .
\end{aligned}
$$

Since we consider numerical integration on the unit hypercube $\Omega=[0,1]^{d} \subset \mathbb{R}^{d}, d \geq 1$, we have

$$
\begin{aligned}
I\left(\phi_{n j}\right) & =\int_{0}^{1} \int_{0}^{1} \cdots \int_{0}^{1} \phi_{n j}\left(x_{1}, x_{2}, \ldots, x_{d}\right) d x_{1} d x_{2} \cdots d x_{d} \\
& =\int_{0}^{1} f_{n}^{*}\left(\alpha\left(x_{1}-x_{1 j}\right)\right) d x_{1} \\
& \times \int_{0}^{1} f_{n}^{*}\left(\alpha\left(x_{2}-x_{2 j}\right)\right) d x_{2} \\
& \cdots \\
& \times \int_{0}^{1} f_{n}^{*}\left(\alpha\left(x_{d}-x_{d j}\right)\right) d x_{d} .
\end{aligned}
$$

Thus, the problem consists in evaluating the $d$ integrals

$$
\begin{aligned}
& \int_{0}^{1} f_{n}^{*}\left(\alpha\left(x_{1}-x_{1 j}\right)\right) d x_{1}, \quad \int_{0}^{1} f_{n}^{*}\left(\alpha\left(x_{2}-x_{2 j}\right)\right) d x_{2}, \\
\ldots \quad & \int_{0}^{1} f_{n}^{*}\left(\alpha\left(x_{d}-x_{d j}\right)\right) d x_{d},
\end{aligned}
$$


and the $h$-th integral, $h=1,2, \ldots, d$, is computed by the relation

$$
\begin{aligned}
& \int_{0}^{1} f_{n}^{*}\left(\alpha\left(x_{h}-x_{h j}\right)\right) d x_{h} \\
= & \frac{1}{\alpha}\left[\Phi_{n}^{*}\left(\alpha\left(1-x_{h j}\right)-\Phi_{n}^{*}\left(\alpha\left(-x_{h j}\right)\right)\right],\right.
\end{aligned}
$$

where the explicit form of $\Phi_{n}^{*}(x)$ is

$\Phi_{n}^{*}(x)=\frac{1}{2^{n} n !} \sum_{k=0}^{n}(-1)^{k}\left(\begin{array}{l}n \\ k\end{array}\right)[x+(n-2 k)]_{+}^{n}$.

Hence, we can approximate a multiple integral by evaluating $d$ simple integrals, whose integrands are given by polynomial functions of degree $n-1$. Note that a numerically equivalent technique, despite computationally less efficient, to evaluate Lobachevsky spline integrals is given in [5].

\subsection{Conditioning and errors}

In this subsection, referring to the error analysis in [5], we focus on stability and accuracy of Lobachevsky spline integration formulas, firstly considering the uniform convergence of Lobachevsky spline to the Gaussian and then discussing the role of two important parameters.

Taking into account the convergence results of Gaussian interpolation (see, e.g., [25]), we can deduce that, for sufficiently regular $g$, the error $\left|I(g)-I\left(F_{n}\right)\right|$ may decrease exponentially as $n \rightarrow \infty$ and $h \rightarrow 0$, where $h$ denotes the so-called fill distance, i.e.

$$
h=\sup _{\mathbf{x} \in \Omega} \min _{\mathbf{x}_{i} \in \mathscr{X}}\left\|\mathbf{x}-\mathbf{x}_{i}\right\|_{2} \text {. }
$$

Moreover, we note that the convergence rate depends on the function $g$, the Lobachevsky spline regularity degree and the form of the domain $\Omega$.

In order to get useful information for numerical implementation, we want to develop a further analysis of the error of the integration formula (6), which can be written by (4) in the form

$I(g) \approx I\left(F_{n}\right)=\langle\mathbf{c}, \mathbf{I}\rangle=\left\langle A^{-1} \mathbf{g}, \mathbf{I}\right\rangle=\langle\mathbf{g}, \mathbf{w}\rangle=\sum_{j=1}^{N} w_{j} g_{j}$,

with

$A \mathbf{w}=\mathbf{I}$,

where $\langle\cdot, \cdot\rangle$ denotes the scalar product in $\mathbb{R}^{d}$, and $\mathbf{I}=\left\{I\left(\phi_{n, j}\right)\right\}_{1 \leq j \leq N}$ is the vector of integrals of Lobachevsky splines. It is clear from (8) that the quantities $\left|w_{j}\right|$ play a central role both in the study of the integration error and in the effect of perturbations in the data values $g_{j}$ [23]. Moreover, the computational trouble caused by the Gaussian ill-conditioning is significantly reduced for Lobachevsky splines, because for small values of $n$ Lobachevsky splines are much better conditioned than Gaussians [4]. As a consequence, the errors obtained by approximating the vector of integrals $\mathbf{I}=\left\{I\left(\phi_{n, j}\right)\right\}_{1<j<N}$ are less influenced than for the Gaussians by the weights $c_{j}$ computed solving the linear system (4).

Now, referring to integration formulas in (8) and (9), we can take into account the presence of errors in the evaluation of the integrals of Lobachevsky splines, i.e.

$\left\{\tilde{I}\left(\phi_{n, j}\right)\right\}=\tilde{\mathbf{I}} \approx \mathbf{I}$,

which generates a vector $\tilde{\mathbf{w}}$ of approximate weights and from (8) the perturbed integration formula

$\tilde{I}\left(F_{n}\right)=\langle\mathbf{c}, \tilde{\mathbf{I}}\rangle=\langle\mathbf{g}, \tilde{\mathbf{w}}\rangle=\sum_{j=1}^{N} \tilde{w}_{j} g_{j}$,

where $\tilde{\mathbf{w}}=A^{-1} \tilde{\mathbf{I}}$.

In error investigation it must also be considered the parameter $\left\|A^{-1}\right\|_{2}$, which measures the sensitivity to perturbations of the interpolation processes based on the Gaussian or Lobachevsky splines. Now, in the literature lower bounds for the smallest eigenvalue of the interpolation matrix $A$ of the Gaussian have been extensively studied (see, e.g., [25]), providing upper bounds for $\left\|A^{-1}\right\|_{2}$ in terms of the so-called separation distance of the nodes

$q=\frac{1}{2} \min _{i \neq j}\left\|\mathbf{x}_{i}-\mathbf{x}_{j}\right\|_{2}$

When $q$ tends to zero, it happens that $\left\|A^{-1}\right\|_{2}$ diverges exponentially and $A$ tends to become singular due to collapsing of two rows. Considering the convergence property of the Lobachevsky splines to the Gaussian, we can reasonably expect that the upper bounds for $\left\|A^{-1}\right\|_{2}$ relating to Lobachevsky splines are closed to Gaussian ones; in fact, numerical tests show that in the case of Lobachevsky splines the values assumed by $\left\|A^{-1}\right\|_{2}$ are quite acceptable and, generally, considerably smaller than those of the Gaussian.

Then, an error estimate of the Lobachevsky spline integration formula (8) may be expressed as follows

$$
\begin{aligned}
|I(g)-\langle\tilde{\mathbf{w}}, \mathbf{g}\rangle| & \leq \operatorname{meas}(\Omega)\left\|g-F_{n}\right\|_{\infty} \\
& +\left\|A^{-1}\right\|_{2}\|\mathbf{I}-\tilde{\mathbf{I}}\|_{2}\|\mathbf{g}\|_{2} .
\end{aligned}
$$

Finally, taking into account the effect of perturbations on the data values, that is, considering the perturbed data

$\left\{\tilde{g}\left(x_{j}\right)\right\}=\tilde{\mathbf{g}} \approx \mathbf{g}$,

we obtain the error estimate

$$
\begin{aligned}
|I(g)-\langle\tilde{\mathbf{w}}, \tilde{\mathbf{g}}\rangle| & \leq\left(\operatorname{meas}(\Omega)+\|\mathbf{w}\|_{1}\right) E_{\mathscr{X}, \phi_{n}}(g) \\
& +\|\mathbf{g}-\tilde{\mathbf{g}}\|_{\infty}\|\mathbf{w}\|_{1}+\left\|A^{-1}\right\|_{2}\|\mathbf{I}-\tilde{\mathbf{I}}\|_{2}\|\tilde{\mathbf{g}}\|_{2},
\end{aligned}
$$

where $E_{\mathscr{X}, \phi_{n}}(g)=\inf _{z \in \operatorname{span}\left(\phi_{n, j}\right)}\|g-z\|_{\infty}($ see [5]). 


\section{Numerical experiments}

In order to test accuracy and stability of the $d$-variate integration formulas obtained by Lobachevsky spline interpolants, we investigate their performances considering some scattered data sets $\mathscr{X} \subseteq \Omega=$ $[0,1]^{d} \subset \mathbb{R}^{d}, 3 \leq d \leq 10$, which are given by Halton points and generated by using the MATLAB program haltonseq.m [16]. Precisely, we firstly consider some sets of Halton points whose size $N$ depend on the dimension $d$, i.e. we take $N=4^{d}$, for $3 \leq d \leq 6$. Then, we keep fixed the point number taking $N=1024$, but varying the dimension $d$, for $6 \leq d \leq 10$. The choice of taking $N$ depending on $d$ is done to analyze the behavior of integration rules maintaining a strict connection between $N$ and $d$. However, since we need to solve a linear system $N \times N$, which (for large $N$ ) turns out to be quite expensive from a computational standpoint, we fix $N$ for $6 \leq d \leq 10$.

Thus, we focus on Lobachevsky spline integration formulas for $n=2,4,6$, that are denoted by L2, L4, L6, respectively, also considering the Gaussian (G) integration formula for comparison. In Figure 1 we show the convergence property of Lobachevsky splines to the Gaussian.

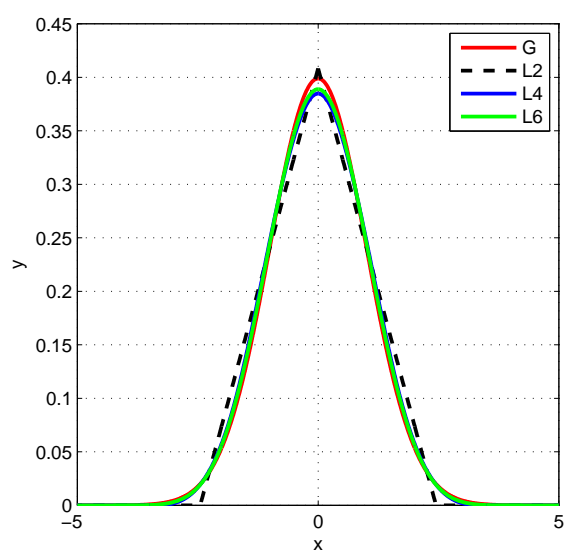

Fig. 1 Example of convergence of Lobachevsky splines to the Gaussian for $d=1$ and $\alpha=1$.

All the results reported below are obtained by using the following $d$-variate test function (see, e.g., [16])

$$
g_{d}(\mathbf{x})=4^{d} \prod_{h=1}^{d} x_{h}\left(1-x_{h}\right), \quad \mathbf{x}=\left(x_{1}, x_{2}, \ldots, x_{d}\right) \in \Omega,
$$

whose exact integral on $\Omega$ is $I\left(g_{d}\right)=(2 / 3)^{d}$. Obviously, other integrands have been tested obtaining a uniform behavior as regard to accuracy and stability.
In Table 1 we show the behavior of Lobachevsky spline integration errors for $3 \leq d \leq 6$ by varying the value of the shape parameter $\alpha \in[1,9]$. Numerical results highlight that the variation of $\alpha$ may greatly influence the quality of approximation results, though the behavior of such errors turns out to be uniform for any $d$. In particular, we remark that the best level of accuracy is reached when $\alpha \in[1,4]$. Moreover, as already remarked in [5] for $d=1,2$, these tests point out reliability and robustness of Lobachevsky spline integration formulas in high dimensions as well.

Using the norm function of MATLAB, in Figures 2-3 we report the values of two crucial quantities considered in Subsection 3.2: the spectral norm $\left\|A^{-1}\right\|_{2}$ and the 1-norm $\|\tilde{\mathbf{w}}\|_{1}$. The former gives us a measure of the absolute conditioning of the considered linear systems, whereas the latter provides useful information on the conditioning of the integration formula.

Thus, for each of the Lobachevsky splines we observe that increasing the shape parameter produces more peaked (or localized) basis functions with the effect of better conditioning, but losing accuracy (and vice versa). Note that, the values of quantities $\left\|A^{-1}\right\|_{2}$ and $\|\tilde{\mathbf{w}}\|_{1}$ strongly depend on the distribution of nodes, while they are independent of function values.

Furthermore, analyzing all the taken tests, we remark that the Lobachevsky splines are comparable in accuracy with the Gaussians, though they are usually much better conditioned than the Gaussians (see Figures 2-3); it holds above all when $\alpha \in[1,4]$ where we have the hightest level of accuracy. Note that there is an exact correspondence between the Lobachevsky and Gaussian shape parameter $\alpha$.

Then, in order to strengthen the effectiveness of the Lobachevsky spline integration formulas, in Table 2 we report the obtained errors by applying a quasi-Monte Carlo method, which in literature is considered as the most practical integration technique in high dimensions. These results confirm once more the goodness of our approach, because for suitable values of the shape parameter $\alpha$ we are able to obtain errors which are lower of one or even two orders of magnitude than those of the quasi-Monte Carlo method.

Finally, in Table 3 we report Lobachevsky spline integration errors with $N=1024$ for $6 \leq d \leq 10$, obtained by varying the value of $\alpha \in[1,5]$ and, for comparison, also considering the results obtained by applying a quasi-Monte Carlo method. These tests (and other ones not reported here for shortness) show that, for a suitable choice of the shape parameter $\alpha$, the Lobachevsky spline integration formulas turn out to be effective for any $d \leq 10$. Conversely, by further increasing $d$, i.e. for $d>10$, we observe a gradual loss of accuracy due to the particular feature of this type of integration formulas, which are expressed as a product of univariate integrals. 
Table 1 Integration errors for $3 \leq d \leq 6$.

\begin{tabular}{|c|c|c|c|c|c|c|}
\hline$d$ & $N$ & $\alpha$ & L2 & L4 & L6 & G \\
\hline \multirow{9}{*}{3} & \multirow{9}{*}{64} & 1 & $5.14 \mathrm{E}-3$ & $9.12 \mathrm{E}-3$ & $2.13 E-3$ & $9.74 \mathrm{E}-4$ \\
\hline & & 2 & $1.15 E-3$ & $2.85 E-3$ & $3.67 \mathrm{E}-3$ & $3.97 \mathrm{E}-3$ \\
\hline & & 3 & $1.21 \mathrm{E}-4$ & $1.47 \mathrm{E}-3$ & $3.10 \mathrm{E}-3$ & $2.73 \mathrm{E}-3$ \\
\hline & & 4 & $3.11 \mathrm{E}-3$ & $3.71 \mathrm{E}-4$ & $3.00 \mathrm{E}-4$ & $3.22 \mathrm{E}-4$ \\
\hline & & 5 & $7.85 \mathrm{E}-3$ & $3.32 \mathrm{E}-4$ & $9.84 \mathrm{E}-4$ & $2.29 \mathrm{E}-3$ \\
\hline & & 6 & $2.15 \mathrm{E}-2$ & $8.44 \mathrm{E}-3$ & $9.60 \mathrm{E}-3$ & $1.27 \mathrm{E}-2$ \\
\hline & & 7 & $4.72 \mathrm{E}-2$ & $2.52 \mathrm{E}-2$ & $2.69 \mathrm{E}-2$ & $3.18 \mathrm{E}-2$ \\
\hline & & 8 & $7.26 \mathrm{E}-2$ & $4.84 \mathrm{E}-2$ & $5.05 \mathrm{E}-2$ & $5.70 \mathrm{E}-2$ \\
\hline & & 9 & $9.86 \mathrm{E}-2$ & $7.49 \mathrm{E}-2$ & $7.76 \mathrm{E}-2$ & $8.53 E-2$ \\
\hline \multirow{9}{*}{4} & \multirow{9}{*}{256} & 1 & $3.24 \mathrm{E}-3$ & $2.30 \mathrm{E}-3$ & $1.52 \mathrm{E}-3$ & $2.75 \mathrm{E}-4$ \\
\hline & & 2 & $2.61 E-3$ & $1.14 \mathrm{E}-3$ & $1.14 \mathrm{E}-3$ & $9.49 \mathrm{E}-5$ \\
\hline & & 3 & $1.36 \mathrm{E}-3$ & $1.26 \mathrm{E}-3$ & $5.95 \mathrm{E}-4$ & $2.60 \mathrm{E}-4$ \\
\hline & & 4 & $1.37 \mathrm{E}-3$ & $1.40 \mathrm{E}-4$ & $1.26 \mathrm{E}-3$ & $1.19 \mathrm{E}-3$ \\
\hline & & 5 & $3.91 \mathrm{E}-3$ & $7.16 \mathrm{E}-4$ & $1.02 \mathrm{E}-3$ & $1.14 \mathrm{E}-4$ \\
\hline & & 6 & $1.20 \mathrm{E}-2$ & $3.67 \mathrm{E}-3$ & $4.21 \mathrm{E}-3$ & $6.51 \mathrm{E}-3$ \\
\hline & & 7 & $2.88 \mathrm{E}-2$ & $1.53 \mathrm{E}-2$ & $1.67 \mathrm{E}-2$ & $2.06 \mathrm{E}-2$ \\
\hline & & 8 & $4.99 \mathrm{E}-2$ & $3.37 \mathrm{E}-2$ & $3.57 \mathrm{E}-2$ & $4.10 \mathrm{E}-2$ \\
\hline & & 9 & $7.27 \mathrm{E}-2$ & $5.61 \mathrm{E}-2$ & $5.84 \mathrm{E}-2$ & $6.45 \mathrm{E}-2$ \\
\hline \multirow{9}{*}{5} & \multirow{9}{*}{1024} & 1 & $2.45 \mathrm{E}-4$ & $1.72 \mathrm{E}-3$ & $1.05 \mathrm{E}-4$ & $1.35 \mathrm{E}-5$ \\
\hline & & 2 & $2.84 \mathrm{E}-5$ & $3.54 \mathrm{E}-4$ & $1.78 \mathrm{E}-5$ & $1.02 \mathrm{E}-4$ \\
\hline & & 3 & $4.59 \mathrm{E}-5$ & $3.13 E-4$ & $2.94 \mathrm{E}-4$ & $2.90 \mathrm{E}-4$ \\
\hline & & 4 & $3.93 E-4$ & $1.10 \mathrm{E}-5$ & $1.80 \mathrm{E}-4$ & $3.73 E-4$ \\
\hline & & 5 & $1.40 \mathrm{E}-4$ & $1.05 \mathrm{E}-3$ & $1.25 \mathrm{E}-3$ & $1.24 \mathrm{E}-3$ \\
\hline & & 6 & $3.09 \mathrm{E}-3$ & $6.94 \mathrm{E}-4$ & $5.04 \mathrm{E}-4$ & $3.48 \mathrm{E}-4$ \\
\hline & & 7 & $1.18 \mathrm{E}-2$ & $4.24 \mathrm{E}-3$ & $5.02 \mathrm{E}-3$ & $7.27 \mathrm{E}-3$ \\
\hline & & 8 & $2.64 \mathrm{E}-2$ & $1.54 \mathrm{E}-2$ & $1.68 \mathrm{E}-2$ & $2.06 \mathrm{E}-2$ \\
\hline & & 9 & $4.53 \mathrm{E}-2$ & $3.19 \mathrm{E}-2$ & $3.38 \mathrm{E}-2$ & $3.88 \mathrm{E}-2$ \\
\hline \multirow{9}{*}{6} & \multirow{9}{*}{4096} & 1 & $2.76 \mathrm{E}-4$ & $3.79 \mathrm{E}-4$ & $4.33 E-4$ & $4.51 \mathrm{E}-5$ \\
\hline & & 2 & $9.40 \mathrm{E}-5$ & $1.12 \mathrm{E}-4$ & $1.24 \mathrm{E}-4$ & $1.48 \mathrm{E}-5$ \\
\hline & & 3 & $1.05 \mathrm{E}-6$ & $7.73 E-5$ & $5.36 \mathrm{E}-5$ & $1.25 \mathrm{E}-4$ \\
\hline & & 4 & $2.64 \mathrm{E}-4$ & $2.16 \mathrm{E}-5$ & $3.24 \mathrm{E}-5$ & $8.33 E-5$ \\
\hline & & 5 & $5.38 \mathrm{E}-4$ & $5.81 \mathrm{E}-4$ & $6.50 \mathrm{E}-4$ & $7.15 E-4$ \\
\hline & & 6 & $7.41 \mathrm{E}-4$ & $9.81 \mathrm{E}-4$ & $9.73 E-4$ & $7.41 \mathrm{E}-4$ \\
\hline & & 7 & $5.18 \mathrm{E}-3$ & $5.63 \mathrm{E}-4$ & $9.05 \mathrm{E}-4$ & $2.07 \mathrm{E}-3$ \\
\hline & & 8 & $1.46 \mathrm{E}-2$ & $6.60 \mathrm{E}-3$ & $7.54 \mathrm{E}-3$ & $1.02 \mathrm{E}-2$ \\
\hline & & 9 & $2.86 \mathrm{E}-2$ & $1.83 \mathrm{E}-2$ & $1.99 \mathrm{E}-2$ & $2.39 \mathrm{E}-2$ \\
\hline
\end{tabular}

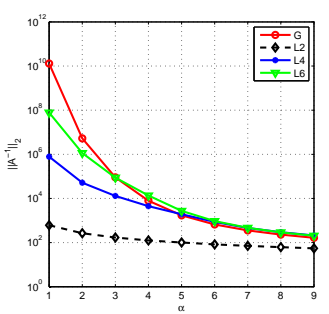

(i) $d=3$

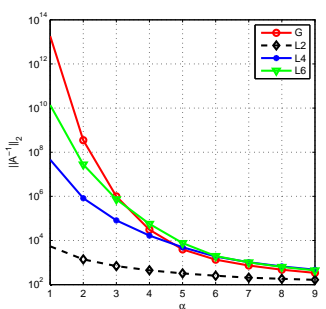

(iii) $d=5$

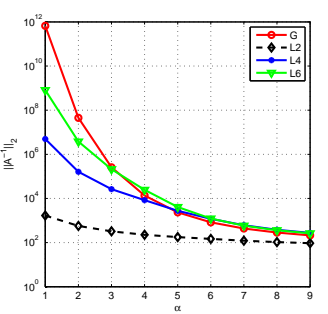

(ii) $d=4$

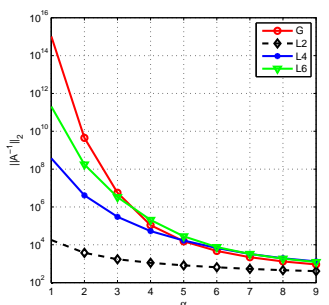

(iv) $d=6$
Fig. 2 Spectral norm of $A^{-1}$ for $3 \leq d \leq 6$.

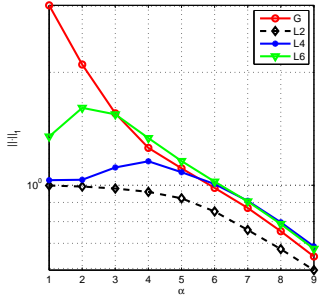

(i) $d=3$

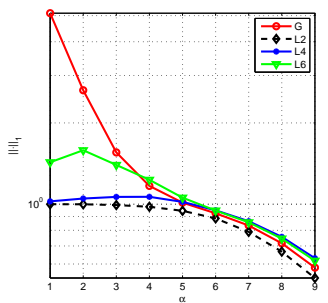

(iii) $d=5$

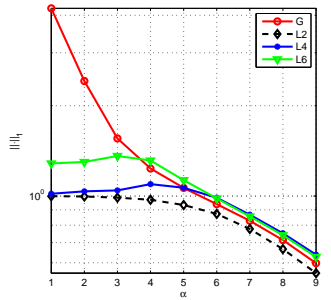

(ii) $d=4$

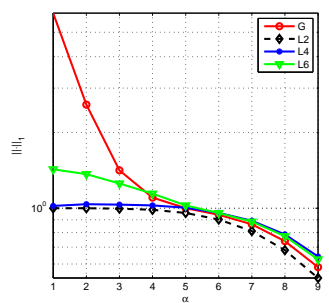

(iv) $d=6$
Fig. 3 1-norm of $\tilde{\mathbf{w}}$ for $3 \leq d \leq 6$.

Table 2 Integration errors of quasi-Monte Carlo for $3 \leq d \leq 6$.

\begin{tabular}{ccc}
\hline$d$ & $N$ & quasi-Monte Carlo \\
\hline 3 & 64 & $1.91 \mathrm{E}-3$ \\
\hline 4 & 256 & $3.43 \mathrm{E}-3$ \\
\hline 5 & 1024 & $5.27 \mathrm{E}-4$ \\
\hline 6 & 4096 & $3.37 \mathrm{E}-4$ \\
\hline
\end{tabular}

\section{Conclusions}

In this paper, thinking of the problem of the curse of dimensionality, we investigated the performance of Lobachevsky spline integration formulas on scattered data in $\mathbb{R}^{d}, 3 \leq d \leq 10$, thus extending the work [5]. In particular, we used the remarkable property of these formulas of being defined in the $d$-variate setting as a product of univariate functions. Moreover, we analyzed accuracy (errors) and stability (conditioning) of such integration formulas as well as those of the Gaussian ones for comparison, referring to two crucial parameters: the spectral norm of the inverses of interpolation matrices and the 1-norm of the computed weight vectors. Numerical experiments confirmed the good performances of Lobachevsky spline integration formulas for $3 \leq d \leq 10$, as already observed in [5] for $d=1,2$. To further check our integration technique, we reported results obtained on the same data point sets by applying a quasi-Monte Carlo method, a standard procedure in multidimensional integration. 
Table 3 Integration errors with $N=1024$ for $6 \leq d \leq 10$.

\begin{tabular}{|c|c|c|c|c|c|}
\hline$d$ & $\alpha$ & $\mathrm{L} 2$ & L4 & L6 & | quasi-Monte Carlo \\
\hline 6 & $\begin{array}{l}1 \\
2 \\
3 \\
4 \\
5\end{array}$ & $\begin{array}{l}5.60 \mathrm{E}-4 \\
3.34 \mathrm{E}-3 \\
1.27 \mathrm{E}-4 \\
7.12 \mathrm{E}-3 \\
1.40 \mathrm{E}-2\end{array}$ & $\begin{array}{l}2.85 \mathrm{E}-2 \\
7.18 \mathrm{E}-3 \\
8.84 \mathrm{E}-4 \\
1.31 \mathrm{E}-2 \\
1.39 \mathrm{E}-2\end{array}$ & $\begin{array}{l}3.73 \mathrm{E}-2 \\
2.98 \mathrm{E}-3 \\
5.06 \mathrm{E}-3 \\
1.26 \mathrm{E}-2 \\
1.20 \mathrm{E}-2\end{array}$ & $2.07 \mathrm{E}-3$ \\
\hline 7 & $\begin{array}{l}1 \\
2 \\
3 \\
4 \\
5\end{array}$ & $\begin{array}{l}1.33 \mathrm{E}-4 \\
1.05 \mathrm{E}-3 \\
1.80 \mathrm{E}-2 \\
1.71 \mathrm{E}-2 \\
9.46 \mathrm{E}-2\end{array}$ & $\begin{array}{l}2.65 \mathrm{E}-2 \\
1.40 \mathrm{E}-3 \\
4.43 \mathrm{E}-3 \\
3.03 \mathrm{E}-2 \\
1.59 \mathrm{E}-2\end{array}$ & $\begin{array}{l}1.04 \mathrm{E}-1 \\
1.53 \mathrm{E}-2 \\
4.90 \mathrm{E}-3 \\
3.04 \mathrm{E}-2 \\
2.48 \mathrm{E}-2\end{array}$ & $8.97 \mathrm{E}-3$ \\
\hline 8 & $\begin{array}{l}1 \\
2 \\
3 \\
4 \\
5\end{array}$ & $\begin{array}{l}8.09 \mathrm{E}-4 \\
5.64 \mathrm{E}-3 \\
5.61 \mathrm{E}-2 \\
1.50 \mathrm{E}-2 \\
2.75 \mathrm{E}-1\end{array}$ & $\begin{array}{l}2.57 \mathrm{E}-2 \\
5.33 \mathrm{E}-2 \\
4.12 \mathrm{E}-3 \\
4.68 \mathrm{E}-2 \\
1.19 \mathrm{E}-1\end{array}$ & $\begin{array}{l}1.22 \mathrm{E}-1 \\
1.55 \mathrm{E}-2 \\
1.11 \mathrm{E}-2 \\
4.59 \mathrm{E}-2 \\
1.38 \mathrm{E}-1\end{array}$ & $1.40 \mathrm{E}-2$ \\
\hline 9 & $\begin{array}{l}1 \\
2 \\
3 \\
4 \\
5\end{array}$ & $\begin{array}{l}3.95 \mathrm{E}-3 \\
2.52 \mathrm{E}-2 \\
1.18 \mathrm{E}-1 \\
4.81 \mathrm{E}-2 \\
5.27 \mathrm{E}-1\end{array}$ & $\begin{array}{l}7.89 E-2 \\
1.05 E-1 \\
3.53 E-2 \\
5.13 E-2 \\
3.33 E-1\end{array}$ & $\begin{array}{l}1.41 \mathrm{E}-2 \\
8.57 \mathrm{E}-2 \\
4.36 \mathrm{E}-2 \\
4.12 \mathrm{E}-2 \\
3.64 \mathrm{E}-1\end{array}$ & $1.81 \mathrm{E}-2$ \\
\hline 10 & $\begin{array}{l}1 \\
2 \\
3 \\
4 \\
5\end{array}$ & $\begin{array}{l}1.05 \mathrm{E}-2 \\
5.19 \mathrm{E}-2 \\
1.74 \mathrm{E}-1 \\
2.07 \mathrm{E}-1 \\
7.40 \mathrm{E}-1\end{array}$ & $\begin{array}{l}1.87 \mathrm{E}-1 \\
1.74 \mathrm{E}-1 \\
7.69 \mathrm{E}-2 \\
3.32 \mathrm{E}-2 \\
5.85 \mathrm{E}-1\end{array}$ & $\begin{array}{l}2.55 \mathrm{E}-1 \\
1.95 \mathrm{E}-1 \\
8.26 \mathrm{E}-2 \\
5.83 \mathrm{E}-2 \\
6.14 \mathrm{E}-1\end{array}$ & $2.55 \mathrm{E}-2$ \\
\hline
\end{tabular}

In a work still in progress, we are going to apply Lobachevsky splines in local methods for fast computation in multivariate and spherical interpolation (see, e.g., $[2,9,10,11])$.

\section{Acknowledgement}

The authors gratefully acknowledge the financial support of the Department of Mathematics "G. Peano", University of Torino, project "Numerical analysis for life sciences" (2012).

\section{References}

[1] G. Allasia, R. Cavoretto, A. De Rossi, B. Quatember, W. Recheis, M. Mayr, and S. Demertzis, In: T.E. Simos et al. (Eds.), Proceedings of the ICNAAM 2010, AIP Conf. Proc., 1281, 716-719 (2010).

[2] G. Allasia, R. Besenghi, R. Cavoretto and A. De Rossi, Appl. Math. Comput., 217, 5949-5966 (2011).

[3] G. Allasia, R. Cavoretto and A. De Rossi, Math. Methods Appl. Sci., 35, 923-934 (2012).

[4] G. Allasia, R. Cavoretto and A. De Rossi, Comput. Appl. Math., 32, 71-87 (2013).

[5] G. Allasia, R. Cavoretto and A. De Rossi, Int. J. Comput. Math., (2013).

[6] G. Allasia, R. Cavoretto and A. De Rossi, Math. Comput. Simulation, submitted for publication, (2013).

[7] A.Yu. Bezhaev, Soviet J. Numer. Anal. Math. Modelling, 6, 95-106 (1991).
[8] R. Brinks, Comput. Appl. Math., 27, 79-92 (2008).

[9] R. Cavoretto and A. De Rossi, J. Comput. Appl. Math., 234, 1505-1521 (2010).

[10] R. Cavoretto and A. De Rossi, Appl. Math. Lett., 25, 12511256 (2012).

[11] R. Cavoretto and A. De Rossi, Math. Methods Appl. Sci., (2013).

[12] R. Cools and D. Laurie (Eds.), Numerical evaluation of integrals, J. Comput. Appl. Math., 112, (1999).

[13] R. Cools, J. Comput. Appl. Math., 149, 1-12 (2002).

[14] R. Cools, D. Huybrechs and D. Nuyen, Int. J. Quantum Chemistry, 109, 1748-1755 (2009).

[15] G. Endrödi, Comput. Phys. Comm., 182, 1307-1314 (2011).

[16] G. E. Fasshauer, Meshfree Approximation Methods with MATLAB, World Scientific Publishing, Singapore, (2007).

[17] B. V. Gnedenko, The Theory of Probability, MIR, Moscow, (1976).

[18] A. R. Krommer and C. W. Ueberhuber, Computational Integration, SIAM, Philadelphia, (1998).

[19] D. Levin, J. Comput. Appl. Math., 112, 181-187 (1999).

[20] A. Punzi, A. Sommariva and M. Vianello, J. Comput. Appl. Math., 221, 430-436 (2008).

[21] A. Rényi, Calcul des Probabilités, Dunod, Paris, (1966).

[22] R. Schürer, Math. Comput. Simulation, 62, 509-517 (2003).

[23] A. Sommariva and M. Vianello, Computing, 76, 295-310 (2006).

[24] A. Sommariva and M. Vianello, Appl. Math. Comput., 183, 1098-1107 (2006).

[25] H. Wendland, Scattered Data Approximation, Cambridge University Press, Cambridge, (2005). 


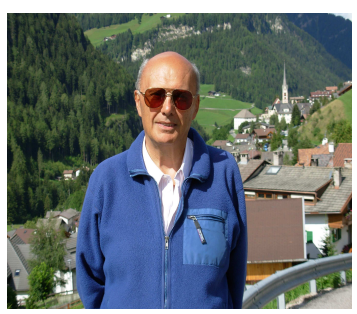

\section{Giampietro Allasia}

is Professor of Numerical Analysis in the Department of Mathematics "Giuseppe Peano" of the University of Torino. His research activity concerns various topics: numerical evaluation of special functions, means and related inequalities, numerical integration formulas, multivariate interpolation on scattered data, history of mathematics. He has published about eighty scientific notes, two books, several encyclopaedic items, and has been editor of the proceedings of four international meetings.

Roberto Cavoretto is a

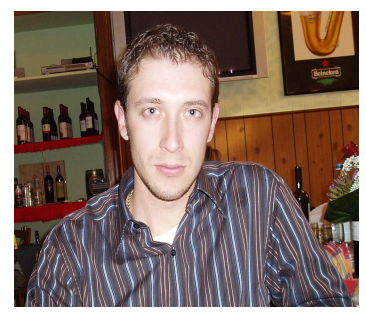

Fixed-Term Research Fellow of Numerical Analysis at the University of Torino, Italy. $\mathrm{He}$ got his Ph.D. degree in Science and High Technology (Mathematics) at the University of Torino in 2010. In the same university he held a post-doc position until 2011. His research activity is mainly focused on topics of numerical analysis and applied mathematics such as: scattered data approximation, spherical interpolation, and applications to image registration. He has published research articles in international refereed journals of applied mathematics.
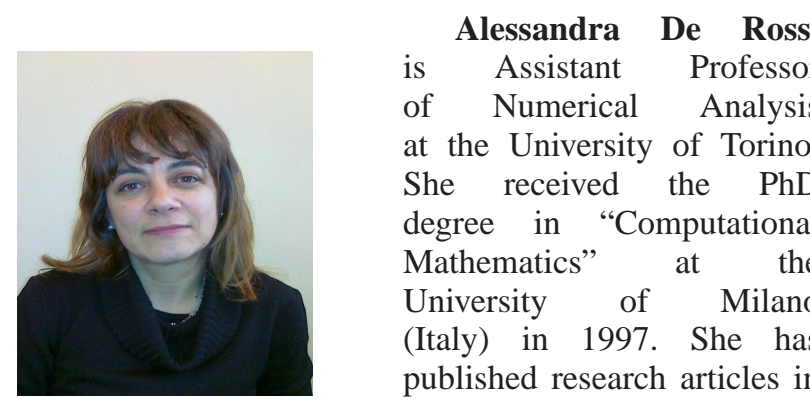

degree in "Computational Mathematics" at the University of Milano (Italy) in 1997. She has published research articles in international journals of pure and applied mathematics. She is referee of several international journals in the frame of numerical analysis and applied mathematics. Her main research interests are: meshfree approximation, fast algorithms for scattered data interpolation, image registration. 